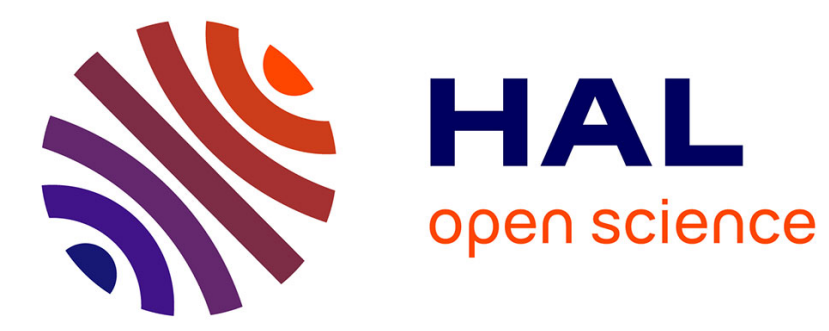

\title{
On the Hougaard subordinated Gaussian Lévy processes
}

Bronius Grigelionis

\section{To cite this version:}

Bronius Grigelionis. On the Hougaard subordinated Gaussian Lévy processes. Statistics and Probability Letters, 2011, 81 (8), pp.998. 10.1016/j.spl.2011.01.020 . hal-00753953

\section{HAL Id: hal-00753953 \\ https://hal.science/hal-00753953}

Submitted on 20 Nov 2012

HAL is a multi-disciplinary open access archive for the deposit and dissemination of scientific research documents, whether they are published or not. The documents may come from teaching and research institutions in France or abroad, or from public or private research centers.
L'archive ouverte pluridisciplinaire HAL, est destinée au dépôt et à la diffusion de documents scientifiques de niveau recherche, publiés ou non, émanant des établissements d'enseignement et de recherche français ou étrangers, des laboratoires publics ou privés. 


\section{Accepted Manuscript}

On the Hougaard subordinated Gaussian Lévy processes

Bronius Grigelionis

PII:

S0167-7152(11)00035-6

DOI:

10.1016/j.spl.2011.01.020

Reference: $\quad$ STAPRO 5895

To appear in: Statistics and Probability Letters

Received date: 26 May 2010

Revised date: 26 January 2011

Accepted date: 26 January 2011

Please cite this article as: Grigelionis, B., On the Hougaard subordinated Gaussian Lévy

processes. Statistics and Probability Letters (2011), doi:10.1016/j.spl.2011.01.020

This is a PDF file of an unedited manuscript that has been accepted for publication. As a service to our customers we are providing this early version of the manuscript. The manuscript will undergo copyediting, typesetting, and review of the resulting proof before it is published in its final form. Please note that during the production process errors may be discovered which could affect the content, and all legal disclaimers that apply to the journal pertain. 


\title{
On the Hougaard subordinated Gaussian Lévy processes
}

\author{
Bronius Grigelionis \\ Institute of Mathematics and Informatics of Vilnius University, Akademijos 4, LT-08663 \\ Vilnius, Lithuania
}

\section{Abstract}

The Hougaard subordinated multivariate Gaussian Lévy processes are characterized. Necessary and sufficient conditions of their selfdecomposability are given and related Ornstein-Uhlenbeck type processes are described.

Keywords: Gaussian Lévy process, Hougaard process, Ornstein-Uhlenbeck type process, self-decomposability, subordination, Thorin class, Thorin measure, Tweedie distribution

\section{INTRODUCTION}

We start with the families of Tweedie or power-variance distributions $\left\{\operatorname{Tw}_{p}(\mu, \lambda), \mu>0, \lambda>0\right\}, p \in \mathbb{R}^{1} \backslash[0,1)$, as exponential dispersion models, characterized by the following properties: for each $\mu>0, \lambda>0$ and given $p$

$$
\begin{gathered}
\int_{\mathbb{R}^{1}} x \operatorname{Tw}_{p}(\mu, \lambda)(d x)=\mu, \\
\int_{\mathbb{R}^{1}}(x-\mu)^{2} \operatorname{Tw}_{p}(\mu, \lambda)(d x)=\lambda^{-1} \mu^{p} ;
\end{gathered}
$$

$\operatorname{Tw}_{0}(\mu, \lambda):=\mathcal{N}\left(\mu, \lambda^{-1}\right), \mu \in \mathbb{R}^{1}, \lambda>0$, are Gaussian distributions.

It is well known (see, e.g., Jørgensen (1997), Vinogradov (2008)) that all these distributions are infinitely divisible and, moreover, for $p \geq 1$ are supported by $\mathbb{R}_{+}:=[0, \infty)$.

A Lévy process $H_{p, \mu, \lambda}=\left\{H_{p, \mu, \lambda}(t), t \geq 0\right\}$ is called the Hougaard process if the probability law $\mathcal{L}\left(H_{p, \mu, \lambda}(1)\right)=\operatorname{Tw}_{p}(\mu, \lambda)$. Thus, if $p \geq 1$, the Hougaard

Email address: broniusgrig@gmail.com (Bronius Grigelionis) 
processes are subordinators. Basing on Grigelionis (2007, 2008), in this paper we characterize the Hougaard subordinated multivariate Gaussian Lévy processes. Necessary and sufficient conditions of their selfdecomposability are given and related Ornstein-Uhlenbeck type processes are described.

\section{The Hougaard subordinated multivariate Gaussian Lévy pro- cesses}

Let $G=\{G(t), t \geq 0\}$ be a Gaussian Lévy process in $\mathbb{R}^{d}$ with the mean vector $a=\mathbb{E} G(1)$ and the covariance matrix $A=\mathbb{E}\left[(G(1)-a)^{\top}(G(1)-a)\right]$, assuming that $|A|:=\operatorname{det} A>0$, where $\top$ means the transposition.

Let

$$
\begin{gathered}
G H_{p}(t)=G\left(H_{p, \mu, \lambda}(t)\right), \\
\mu_{p}^{t}=\mathcal{L}\left(G H_{p}(t)\right), \quad t \geq 0, \quad p \geq 1,
\end{gathered}
$$

assuming that processes $G$ and $H_{p, \mu, \lambda}$ are independent,

$$
G_{a, A}(B)=\int_{B} g_{a, A}(x) d x, \quad B \in \mathcal{B}\left(\mathbb{R}^{d}\right),
$$

where

$$
g_{a, A}(x)=\frac{1}{\sqrt{|A|}(2 \pi)^{d / 2}} \exp \left\{-\frac{1}{2}\left((x-a) A^{-1},(x-a)\right)\right\}, \quad x \in \mathbb{R}^{d} .
$$

Theorem 1. (i) $G H_{1}$ is a Lévy process with the triplet of Lévy characteristics $\left(a_{1}, 0, \nu_{1}\right)$ and

$$
\mu_{1}^{t}(B)=\sum_{k=0}^{\infty} G_{\lambda^{-1} a k, \lambda^{-1} A k}(B) e^{-\mu \lambda t} \frac{(\mu \lambda t)^{k}}{k !}, \quad t \geq 0
$$

where

$$
a_{1}=\mu \lambda \int_{|x| \leq 1} x G_{\lambda^{-1} a, \lambda^{-1} A}(d x)
$$

and

$$
\nu_{1}(B)=\mu \lambda G_{\lambda^{-1} a, \lambda^{-1} A}(B), \quad B \in \mathcal{B}\left(\mathbb{R}^{d} \backslash\{0\}\right) ;
$$


(ii) for $p>1, G H_{p}$ is a Lévy process with the triplet $\left(a_{p}, 0, \nu_{p}\right)$ of Lévy characteristics and

$$
\mu_{p}^{t}(B)=\int_{0}^{\infty} G_{s a, s A}(B) \operatorname{Tw}_{p}\left(\mu t, \lambda t^{p-1}\right)(d s), \quad t \geq 0
$$

where

$$
\begin{gathered}
a_{p}=\int_{|x| \leq 1} x g_{p}(x) d x, \quad \nu_{p}(B)=\int_{B} g_{p}(x) d x, \quad B \in \mathcal{B}\left(\mathbb{R}^{d} \backslash\{0\}\right), \\
g_{p}(x)=\frac{2 c_{p, \lambda} \exp \left\{\left(x A^{-1}, a\right)\right\}}{\sqrt{|A|}(2 \pi)^{d / 2}}\left(\frac{\frac{2 \lambda \mu^{1-p}}{p-1}+\left(a A^{-1}, a\right)}{\left(x A^{-1}, x\right)}\right)^{\frac{p-2}{2(p-1)}+d / 4} \cdot \\
\cdot K_{\frac{p-2}{p-1}+\frac{d}{2}}\left(\left[\left(\frac{2 \lambda \mu^{1-p}}{p-1}+\left(a A^{-1}, a\right)\right)\left(x A^{-1}, x\right)\right]^{\frac{1}{2}}\right), \\
c_{p, \lambda}=\frac{\lambda^{\frac{1}{p-1}}}{\Gamma\left(\frac{p}{p-1}\right)(p-1)^{\frac{p}{p-1}}}
\end{gathered}
$$

and

$$
K_{\nu}(z)=\frac{1}{2}\left(\frac{z}{2}\right)^{\nu} \int_{0}^{\infty} t^{-\nu-1} \exp \left\{-t-\frac{z^{2}}{4 t}\right\} d t, \quad z, \nu \in \mathbb{R}^{1}
$$

is the modified Bessel function of the third kind.

Proof. Let $p \geq 1$. It is known (see Jorgensen and Martínez (1996)) that $H_{p, \mu, \lambda}$ is a Lévy subordinator with zero drift. Let $\rho_{p}$ be its Lévy measure. From Theorem 30.1 of Sato (1999) it follows that $G H_{p}$ is a Lévy process with

$$
\mu_{p}^{t}(B)=\int_{0}^{\infty} G_{s a, s A}(B) \mathcal{L}\left(H_{p, \mu, \lambda}(t)\right)(d s)
$$


and with the triplet of Lévy characteristics $\left(a_{p}, 0, \nu_{p}\right)$, where

$$
a_{p}=\int_{0}^{\infty} \int_{|x| \leq 1} x g_{s a, s A}(x) d x \rho_{p}(d s)
$$

and the Lévy measure $\nu_{p}$ is given by

$$
\nu_{p}(B)=\int_{0}^{\infty} G_{s a, s A}(B) \rho_{p}(d s), \quad B \in \mathcal{B}\left(R^{d} \backslash\{0\}\right) .
$$

If $p=1$, then $\rho_{1}(d s)=\mu \lambda \varepsilon_{\lambda^{-1}}$, where $\varepsilon_{\Delta}$ is the Dirac measure, implying that $T_{W_{1}}(\mu, \lambda)$ is the scaled Poisson distribution, i.e.,

$$
T w_{1}(\mu, \lambda)\left(\left\{\lambda^{-1} k\right\}\right)=e^{-\mu \lambda} \frac{(\mu \lambda)^{k}}{k !}, \quad k=0,1, \ldots
$$

and showing formulas (3) and (4). Since the Hougaard process has the following scaling property

$$
\mathcal{L}\left(H_{p, \mu, \lambda}(t)\right)=T w_{p}\left(\mu t, \lambda t^{p-1}\right),
$$

we also obtain (2) and (5).

If $p>1$, then

$$
\rho_{p}(d x)=c_{p, \lambda} x^{\frac{3-2 p}{p-1}} \exp \left\{-\frac{\lambda \mu^{1-p}}{p-1} x\right\} d x .
$$

Now (6) and (7) are consequences of the following formula: for all $\alpha, \beta \geq 0$, $\nu \in R^{1}$ and $x \in R^{d}$

$$
\begin{aligned}
& \int_{0}^{\infty} g_{s a, s A}(x) s^{\nu} e^{-a s-\beta / s} d s \\
& =\frac{2 \exp \left\{\left(x A^{-1}, \alpha\right)\right\}}{\sqrt{|A|}(2 \pi)^{d / 2}}\left(\frac{2 \alpha+\left(a A^{-1}, \alpha\right)}{2 \beta+\left(x A^{-1}, x\right)}\right)^{\frac{-\nu}{2}+\frac{d}{4}-\frac{1}{2}} \cdot \\
& \cdot K_{\nu-d / 2+1}\left(\left[\left(2 \alpha+\left(a A^{-1} a\right)\right)\left(2 \beta+\left(x A^{-1}, x\right)\right)\right]^{1 / 2}\right) .
\end{aligned}
$$


Indeed, from (9) we derive that for all $\gamma, \delta>0$

$$
\int_{0}^{\infty} t^{-\nu-1} e^{-\gamma t-\delta / t} d t=2\left(\frac{\gamma}{\delta}\right)^{\frac{\nu}{2}} K_{\nu}(2 \sqrt{\gamma \delta}),
$$

having in mind that $K_{\nu}(z)=K_{-\nu}(z)$ (see, e.g., Watson (1958)). Now (11) follows from (1) and (12) after elementary calculations. Formulas (5), (6) easily follow from (11) and Theorem 30.1 of Sato (1999).

Remark 2. If $p=\frac{3}{2}$ (the compound Poisson-exponential distribution case), we have (see Vinogradov (2007))

$$
\begin{aligned}
\mathrm{Tw}_{3 / 2}(\mu t, \lambda \sqrt{t})(C) & =e^{-2 \lambda \sqrt{\mu} t} \varepsilon_{0}(C)+ \\
& +\int_{C} \frac{2 \lambda \sqrt{t}}{\sqrt{s}} e^{-2 \frac{\lambda}{\sqrt{\mu}}(s+\mu t)} I_{1}(4 \lambda \sqrt{s t}) d s, \quad C \in \mathcal{B}\left(\mathbb{R}_{+}\right),
\end{aligned}
$$

where $I_{\nu}(z)$ is the modified Bessel function of the first kind, i.e.,

$$
I_{\nu}(z)=\left(\frac{z}{2}\right)^{\nu} \sum_{k=0}^{\infty} \frac{\left(\frac{z}{2}\right)^{2 k}}{k ! \Gamma(\nu+k+1)}, \quad \nu \geq-1,
$$

and $\Gamma(z)$ is the Euler's gamma function.

From (10), (13) and (14) it follows that

$$
\begin{gathered}
\mu_{3 / 2}^{t}(B)=e^{-2 \lambda \sqrt{\mu} t} \varepsilon_{0}(B)+\frac{4 \lambda^{2} t e^{2 \lambda \sqrt{\mu} t}}{\sqrt{|A|}(2 \pi)^{d / 2}}\left(\frac{4 \lambda}{\sqrt{\mu}}+\left(a A^{-1}, a\right)\right)^{d / 4-1 / 2} \cdot \\
\cdot \sum_{k=0}^{\infty} \int_{B} \frac{\left(4 \lambda^{2} t\left(x A^{-1}, x\right)^{1 / 2}\right)^{k}}{k !(k+1) !} \cdot \\
\cdot K_{k-d / 2+1}\left(\left[\left(\frac{4 \lambda}{\sqrt{\mu}}+\left(a A^{-1}, a\right)\right)\left(x A^{-1}, x\right)\right]^{1 / 2}\right) d x, \quad t \geq 0, \quad B \in \mathcal{B}\left(\mathbb{R}^{d}\right) .
\end{gathered}
$$

Remark 3. If $p=2$ (the gamma distribution case), we have

$$
\mathrm{Tw}_{2}(\mu t, \lambda t)(C)=\int_{C} \frac{\left(\frac{\lambda}{\mu}\right)^{\lambda t} s^{\lambda t-1}}{\Gamma(\lambda t)} e^{-\frac{\lambda}{\mu} s} d s, \quad C \in \mathcal{B}\left(\mathbb{R}_{+}\right)
$$


implying that

$$
\begin{aligned}
& \mu_{2}^{t}(B)=\frac{2\left(\frac{\lambda}{\mu}\right)^{\lambda t}}{\sqrt{|A|}(2 \pi)^{d / 2} \Gamma(\lambda t)} \int_{B} \exp \left\{\left(x A^{-1}, a\right)\right\}\left(\frac{\frac{2 \lambda}{\mu}+\left(a A^{-1}, a\right)}{\left(x A^{-1}, x\right)}\right)^{-\lambda t / 2+d / 4} . \\
& \cdot K_{\lambda t-d / 2}\left(\left[\left(\frac{2 \lambda}{\mu}+\left(a A^{-1}, a\right)\right)\left(x A^{-1}, x\right)\right]^{1 / 2}\right) d x, \quad t \geq 0, \quad B \in \mathcal{B}\left(\mathbb{R}^{d}\right) .
\end{aligned}
$$

Remark 4. If $p=3$ (the inverse Gaussian distribution case), we have

$$
\mathrm{Tw}_{3}\left(\mu t, \lambda t^{2}\right)(C)=\int_{C} \sqrt{\frac{\lambda}{2 \pi}} t e^{\frac{\lambda}{\mu} t} s^{-\frac{3}{2}} e^{-\frac{\lambda s}{2 \mu^{2}}-\frac{\lambda t^{2}}{2 s}} d s, \quad t>0, \quad C \in \mathcal{B}\left(\mathbb{R}^{d}\right),
$$

implying that

$$
\begin{array}{rl}
\mu_{3}^{t}(B)= & \frac{\sqrt{\lambda} t e^{\frac{\lambda}{\mu} t}}{\sqrt{|A|}(2 \pi)^{\frac{d+1}{2}}} \int_{B}\left(\frac{\frac{\lambda}{\mu^{2}}+\left(a A^{-1}, a\right)}{\lambda t^{2}+\left(x A^{-1}, x\right)}\right)^{\frac{d+1}{4}} . \\
& \cdot K_{\frac{d+1}{2}}\left(\left[\left(\frac{\lambda}{\mu^{2}}+\left(a A^{-1}, a\right)\right)\left(\lambda t^{2}+\left(x A^{-1}, x\right)\right)\right]^{1 / 2}\right) d x, \\
t & t>0, B \in \mathcal{B}\left(\mathbb{R}^{d}\right) .
\end{array}
$$

\section{Selfdecomposability of subordinated processes}

A Lévy process $X=\{X(t), t \geq 0\}$ in $\mathbb{R}^{d}$ with characteristic function $\hat{\mu}(z):=\mathbb{E} \exp \{i(z, X(1))\}, z \in \mathbb{R}^{d}$, is said to be selfdecomposable or of class $L\left(\mathbb{R}^{d}\right)$ if, for any $b>1$, there is a characteristic function $\hat{\mu}_{b}$ such that $\hat{\mu}(z)=$ $\hat{\mu}\left(b^{-1} z\right) \hat{\mu}_{b}(z), z \in \mathbb{R}^{d}$. If the Lévy measure $\nu$ of $X$ equals

$$
\nu(B)=\int_{B} g(x) d x, \quad B \in \mathcal{B}\left(\mathbb{R}^{d} \backslash\{0\}\right),
$$

then, according to Proposition 1 in Grigelionis (2007), $X$ is selfdecomposable if and only if for a.e. $\xi \in S^{d-1}:=\left\{x \in \mathbb{R}^{d}:|x|=1\right\}$ with respect to the surface Lebesgue measure on $S^{d-1}$ the function $k(\xi, r):=r^{d} g(r \xi), r>0$, is decreasing. 
Theorem 5. The Hougaard subordinated Gaussian Lévy process $G H_{p}$ is selfdecomposable if and only if $p \geq 2, d=1$ or $p \geq 2, d \geq 2$ and $a=0$.

Proof. Let $p=1$. From (4) we find that

$$
\nu_{1}(B)=\int_{B} \mu \lambda g_{\lambda^{-1} a, \lambda^{-1} A}(x) d x, \quad B \in \mathcal{B}\left(\mathbb{R}^{d} \backslash\{0\}\right) .
$$

Because, obviously, the function

$$
k_{1}(\xi, r)=\mu \lambda r^{d} g_{\lambda^{-1} a, \lambda^{-1} A}(r \xi), \quad r>0,
$$

is not decreasing for each $\xi \in S^{d-1}$, the process $G H_{1}$ is not selfdecomposable.

Now let $p>1$. From (10) it follows that for each $\mu>0, \lambda>0, H_{p, \mu, \lambda}$ is subordinator of the Thorin class $T^{\left(\frac{1}{p-1}\right)}\left(\mathbb{R}_{+}\right)$(see Grigelionis (2008)) with the Thorin measure

$$
Q_{\frac{1}{p-1}}(d v)=c_{p, \lambda} \exp \left\{-\frac{\lambda \mu^{1-p}}{p-1} v\right\} d v .
$$

If $1<p<2$, from (15) and Proposition 4 (v) of Grigelionis (2008), we find that the processes $G H_{p}$ are not selfdecomposable.

If $p \geq 2$, then $T^{\left(\frac{1}{p-1}\right)}\left(\mathbb{R}_{+}\right) \subseteq T^{(1)}\left(\mathbb{R}_{+}\right)$and the assertion of the theorem follows from Proposition 4 (i) of Grigelionis (2008).

Finally, we describe the background driving Lévy processes

$$
Z_{p}^{(c)}=\left\{Z_{p}^{(c)}(t), t \geq 0\right\}, \quad c>0
$$

such that the Ornstein-Uhlenbeck type processes

$$
X_{p}(t)=e^{-c t} X_{p}(0)+\int_{0}^{t} e^{-c(t-s)} d Z_{p}^{(c)}(s), \quad t \geq 0, \quad c>0
$$

are strictly stationary, provided $\mathcal{L}\left(X_{p}(0)\right)=\mathcal{L}\left(G H_{p}(1)\right), G H_{p}$ is selfdecomposable and $Z_{p}^{(c)}$ is independent of $X_{p}(0)$. 
Theorem 6. If $p \geq 2, d=1$ or $p \geq 2, d \geq 2, a=0$ and the background driving Lévy process $Z_{p}^{(c)}$ has the triplet $\left(a_{p}^{(c)}, 0, \nu_{p}^{(c)}\right)$ of Lévy characteristics, where

$$
a_{p}^{(c)}=\int_{|x| \leq 1} x g_{p}^{(c)}(x) d x, \quad \nu_{p}^{(c)}(B)=\int_{B} g_{p}^{(c)}(x) d x, \quad B \in \mathcal{B}\left(\mathbb{R}^{d} \backslash\{0\}\right),
$$

and

$$
g_{p}^{(c)}(x)=-\left.\frac{d}{d r}\left(c r^{d} g_{p}(r \xi)\right)\right|_{r \xi=x},
$$

then $X_{p}$ in (16) is strictly stationary.

Proof. The statement of Theorem 6 follows directly from Proposition 1 in Grigelionis (2007), and Theorem 17.5 in Sato (1999).

Remark 7. If $p \geq 2, d \geq 2$ and $a=0$, from (7) we have

$$
\begin{aligned}
r^{d} g_{p}(r \xi)=\frac{2 c_{p, \lambda}}{\sqrt{|A|}(2 \pi)^{d / 2}}\left(\frac{2 \lambda \mu^{1-p}}{r^{2}\left(\xi A^{-1}, \xi\right)}\right)^{\frac{p-2}{2(p-1)}+\frac{d}{4}} & \cdot \\
\cdot K_{\frac{p-2}{p-1}+\frac{d}{2}} & \left(\left[\frac{2 r^{2} \lambda \mu^{1-p}}{p-1}\left(\xi A^{-1}, \xi\right)\right]^{1 / 2}\right),
\end{aligned}
$$

and, using the equality (see, e.g., Watson (1958))

$$
K_{\nu}^{\prime}(z)=-\left(K_{\nu-1}(z)+\frac{\nu}{2} K_{\nu}(z)\right)
$$

we find that

$$
g_{p}^{(c)}(x)=C_{p}\left(x A^{-1}, x\right)^{\frac{2 p-3}{p-1}+\frac{d}{4}} K_{\frac{1}{p-1}-\frac{d}{2}}\left(\left[\frac{2 \lambda \mu^{1-p}}{p-1}\left(x A^{-1}, x\right)\right]^{1 / 2}\right),
$$

where

$$
C_{p}=\frac{2 c c_{p, \lambda}\left(2 \lambda \mu^{1-p}\right)^{\frac{2 p-3}{2(p-1)}+\frac{d}{4}}}{\sqrt{|A|}(2 \pi)^{d / 2} \sqrt{p-1}} .
$$

Acknowledgement. The author is grateful to the anonymous referee for useful remarks. 


\section{References}

Grigelionis, B., 2007. On subordinated multivariate Gaussian Lévy processes. Acta Appl. Math. 96 (1-3), 233-246.

Grigelionis, B., 2008. Thorin classes of Lévy processes and their transforms. Lith. Math. J. 48 (3), 294-315.

Jørgensen, B., 1997. The theory of dispersion models. Vol. 76 of Monographs on Statistics and Applied Probability. Chapman \& Hall, London.

Jørgensen, B., Martínez, J. R., 1996. The Lévy-Khinchine representation of the Tweedie class. Braz. J. Probab. Stat. 10 (2), 225-233.

Sato, K.-i., 1999. Lévy processes and infinitely divisible distributions. Vol. 68 of Cambridge Studies in Advanced Mathematics. Cambridge University Press, Cambridge.

Vinogradov, V., 2007. On infinitely divisible exponential dispersion model related to Poisson-exponential distribution. Comm. Statist. Theory Methods 36 (1-4), 253-263.

Vinogradov, V., 2008. Properties of certain Lévy and geometric Lévy processes. Commun. Stoch. Anal. 2 (2), 193-208.

Watson, G. N., 1958. A treatise on the theory of Bessel functions. Cambridge University Press, Cambridge. 